DNA methylation in childhood asthma : an epigenome-wide meta-analysis

\author{
Xu, Cheng-Jian
}

2018-05

Xu , C-J , Soderhall , C, Bustamante , M , Baiz , N, Gruzieva, O , Gehring , U , Mason , D , Chatzi , L , Basterrechea , M , Llop , S , Torrent , M , Forastiere , F , Fantini , M P , Carlsen , K C L , Haahtela , T, Morin , A, Kerkhof , M , Merid , S K, van Rijkom , B , Jankipersadsing , S A , Bonder , M J , Ballereau , S , Vermeulen, C J , Aguirre-Gamboa , R, de Jongste , J C , Smit , H A , Kumar , A, Pershagen, G, Guerra , S, Garcia-Aymerich , J , Greco , D , Reinius , L, McEachan , R R C , Azad , R, Hovland , V, Mowinckel , P , Alenius , H , Fyhrquist, N , Lemonnier , N, Pellet , J Auffray , C , van der Vlies , P , van Diemen , C C , $\mathrm{Li}, \mathrm{Y}$, Wijmenga , C , Netea, M G, Moffatt, M F , Cookson, W O C M , Anto , J M \& Kere , J 2018 , ' DNA methylation in childhood asthma : an epigenome-wide meta-analysis ', The Lancet respiratory medicine , vol. 6 , no. 5 , pp. 379-388 . https://doi.org/10.1016/S2213-2600(18)30052-3

http://hdl.handle.net/10138/301557

https://doi.org/10.1016/S2213-2600(18)30052-3

unspecified

publishedVersion

Downloaded from Helda, University of Helsinki institutional repository.

This is an electronic reprint of the original article.

This reprint may differ from the original in pagination and typographic detail.

Please cite the original version. 


\title{
DNA methylation in childhood asthma: an epigenome-wide meta-analysis
}

\author{
Cheng-Jian Xu, Cilla Söderhäll, Mariona Bustamante, Nour Baïz, Olena Gruzieva, Ulrike Gehring, Dan Mason, Leda Chatzi, Mikel Basterrechea, \\ Sabrina Llop, Maties Torrent, Francesco Forastiere, Maria Pia Fantini, Karin C Lødrup Carlsen, Tari Haahtela, Andréanne Morin, Marjan Kerkhof, \\ Simon Kebede Merid, Bianca van Rijkom, Soesma A Jankipersadsing, Marc Jan Bonder, Stephane Ballereau, Cornelis JVermeulen, Raul Aguirre-Gamboa, \\ Johan C de Jongste, Henriette A Smit, Ashish Kumar, Göran Pershagen, Stefano Guerra, Judith Garcia-Aymerich, Dario Greco, Lovisa Reinius, \\ Rosemary R CMcEachan, Raf Azad, Vegard Hovland, Petter Mowinckel, Harri Alenius, Nanna Fyhrquist, Nathanaël Lemonnier, Johann Pellet, \\ Charles Auffray, the BIOS Consortium, Pieter van der Vlies, Cleo C van Diemen, Yang Li, Cisca Wijmenga, Mihai G Netea, Miriam F Moffatt, \\ William O C M Cookson, Josep M Anto, Jean Bousquet, Tiina Laatikainen, Catherine Laprise, Kai-Håkon Carlsen, Davide Gori, Daniela Porta, \\ Carmen Iñiguez, Jose Ramon Bilbao, Manolis Kogevinas, John Wright, Bert Brunekreef, Juha Kere, Martijn C Nawijn, Isabella Annesi-Maesano, \\ Jordi Sunyer, Erik Melén*, Gerard H Koppelman*
}

\section{Summary}

Background DNA methylation profiles associated with childhood asthma might provide novel insights into disease pathogenesis. We did an epigenome-wide association study to assess methylation profiles associated with childhood asthma.

Methods We did a large-scale epigenome-wide association study (EWAS) within the Mechanisms of the Development of ALLergy (MeDALL) project. We examined epigenome-wide methylation using Illumina Infinium Human Methylation450 BeadChips (450K) in whole blood in 207 children with asthma and 610 controls at age 4-5 years, and 185 children with asthma and 546 controls at age 8 years using a cross-sectional case-control design. After identification of differentially methylated CpG sites in the discovery analysis, we did a validation study in children (4-16 years; 247 cases and 2949 controls) from six additional European cohorts and meta-analysed the results. We next investigated whether replicated CpG sites in cord blood predict later asthma in 1316 children. We subsequently investigated celltype-specific methylation of the identified $\mathrm{CpG}$ sites in eosinophils and respiratory epithelial cells and their related gene-expression signatures. We studied cell-type specificity of the asthma association of the replicated $\mathrm{CpG}$ sites in 455 respiratory epithelial cell samples, collected by nasal brushing of 16-year-old children as well as in DNA isolated from blood eosinophils (16 with asthma, eight controls [age 2-56 years]) and compared this with whole-blood DNA samples of 74 individuals with asthma and 93 controls (age 1-79 years). Whole-blood transcriptional profiles associated with replicated CpG sites were annotated using RNA-seq data of subsets of peripheral blood mononuclear cells sorted by fluorescence-activated cell sorting.

Findings 27 methylated CpG sites were identified in the discovery analysis. 14 of these $\mathrm{CpG}$ sites were replicated and passed genome-wide significance $\left(p<1 \cdot 14 \times 10^{-7}\right)$ after meta-analysis. Consistently lower methylation levels were observed at all associated loci across childhood from age 4 to 16 years in participants with asthma, but not in cord blood at birth. All $14 \mathrm{CpG}$ sites were significantly associated with asthma in the second replication study using wholeblood DNA, and were strongly associated with asthma in purified eosinophils. Whole-blood transcriptional signatures associated with these CpG sites indicated increased activation of eosinophils, effector and memory CD8 T cells and natural killer cells, and reduced number of naive $\mathrm{T}$ cells. Five of the $14 \mathrm{CpG}$ sites were associated with asthma in respiratory epithelial cells, indicating cross-tissue epigenetic effects.

Interpretation Reduced whole-blood DNA methylation at $14 \mathrm{CpG}$ sites acquired after birth was strongly associated with childhood asthma. These CpG sites and their associated transcriptional profiles indicate activation of eosinophils and cytotoxic $\mathrm{T}$ cells in childhood asthma. Our findings merit further investigations of the role of epigenetics in a clinical context.

Funding EU and the Seventh Framework Programme (the MeDALL project).

\section{Introduction}

Asthma is a heterogeneous chronic inflammatory airway disease, characterised by variable respiratory symptoms and reversible airflow limitation. Worldwide, more than 300 million people have asthma, with substantial morbidity, reduced quality of life (of patients and their families), and substantial health-care costs. ${ }^{1}$ Asthma originates predominantly in early childhood. ${ }^{2}$ According to twin studies, about $50-60 \%$ of asthma susceptibility is explained by genetic factors. ${ }^{3}$ Large-scale genome-wide association studies (GWAS) have uncovered several genetic variants related to asthma, some of which are specific to childhood-onset asthma. ${ }^{45}$ The dramatic increase in asthma prevalence in the past half-century
Lancet Respir Med 2018; 6: 379-88 Published Online February 23, 2018 http://dx.doi.org/10.1016 S2213-2600(18)30052-3 See Comment page 322 *Contributed equally Department of Pulmonology (C) Xu PhD,

SA Jankipersadsing MSC, CJVermeulen PhD), GRIAC research institute Groningen (C) Xu, C J Vermeulen, M Kerkhof PhD, M C Nawijn PhD, Prof G H Koppelman PhD), Department of Genetics ( $C)$ Xu, $B$ van Rijkom BSc, SA Jankipersadsing, MJ Bonder PhD, R Aguirre-Gamboa MSc, $P$ van der Vlies BSC, C C van Diemen PhD, Y Li PhD, Prof (Wijmenga PhD), Department of Pathology and Medical Biology, Experimental Pulmonology and Inflammation Research (M C Nawijn), and Department of Pediatric Pulmonology and Pediatric Allergy, Beatrix Children's Hospital (Prof G H Koppelman), University Medical Center Groningen, University of Groningen, Groningen, the Netherlands; Department of Women's and Children's Health (C Söderhäll PhD), Department of Biosciences and Nutrition (C Söderhäll, L Reinius PhD, Prof J Kere PhD), and Institute of Environmental Medicine (O Gruzieva PhD, A Kumar MSc, S Kebede Merid MSC, Prof G Pershagen PhD, H Alenius PhD, N Fyhrquist PhD, E Melén PhD), Karolinska Institutet, Stockholm, Sweden; ISGlobal, Centre for Research in 
Environmental Epidemiology (M Bustamante PhD, Prof S Guerra MD, J Garcia-Aymerich PhD, ProfJ M Anto PhD, $M$ Kogevinas PhD, Prof J Sunyer PhD) and Centre for Genomic Regulation (M Bustamante), the Barcelona Institute of Science and Technology, Barcelona, Spain; Universitat Pompeu Fabra, Barcelona, Spain (M Bustamante, J Garcia-Aymerich, Prof J M Anto, Prof J Sunyer); CIBER Epidemiología y Salud Pública (CIBERESP), Madrid, Spain (M Bustamante, M Basterrechea MD, S Llop PhD, J Garcia-Aymerich, Prof J M Anto,

C Iñiguez PhD, M Kogevinas, Prof J Sunyer); Epidemiology of

Allergic and Respiratory Diseases Department (EPAR), Sorbonne Université, INSERM,

Pierre Louis Institute of

Epidemiology and Public

Health, Saint-Antoine Medical School, Paris, France (N Baïz PhD,

Prof I Annesi-Maesano PhD); Institute for Risk Assessment Sciences (U Gehring PhD, Prof B Brunekreef $\mathrm{PhD}$ ) and Julius Center for Health Sciences and Primary Care, University Medical Center Utrecht (H A Smit PhD, Prof B Brunekreef), Utrecht University, the Netherlands; Bradford Institute for Health

Research, Bradford Teaching Hospitals NHS Foundation Trust, Bradford, UK (D Mason PhD,

R R C McEachan PhD,

R Azad FRCP, J Wright FRCP); Department of Preventive Medicine, Keck School of

Medicine, University of Southern California, LA, USA (LChatzi MD); Department of

Social Medicine, Faculty of Medicine, University of Crete, Heraklion, Crete, Greece (LChatzi); Department of Genetics and Cell Biology, Faculty of Health, Medicine and Life Sciences, Maastricht

University, Maastricht, the Netherlands (L Chatzi); Health Research Institute Biodonostia, San Sebastián, Spain (M Basterrechea); Public Health Department of Gipuzkoa, San Sebastián, Spain (M Basterrechea); Epidemiology and Environmental Health Joint Research Unit, FISABIO-Universitat Jaume

\section{Research in context}

\section{Evidence before this study}

We searched for articles in PubMed published in English up to Oct 17, 2017, with the search terms "epigenetics", "asthma", and "children", and selected key candidate and epigenome-wide studies to be reviewed in more detail. We also searched for relevant references in review articles from experts in the field. We found evidence that epigenetic changes, including DNA methylation CpG sites, non-coding RNAs, and microRNAs had been associated with asthma in previous studies. However, it was largely unknown which DNA methylation sites were robustly associated with asthma across the genome and whether age-specific patterns can be observed in children from different countries with varying environmental exposures and lifestyles.

\section{Added value of this study}

To our knowledge, this is the largest epigenetics study on asthma to date, using data from more than 5000 children in ten cohorts. By applying stringent genome-wide significance criteria $\left(p<1.14 \times 10^{-7}\right)$, our study identified reduced DNA methylation levels in $14 \mathrm{CpG}$ sites to be associated with asthma across childhood from ages 4 to 16 years, but not at birth. Asthma associated whole blood DNA methylation profiles was strongly driven by lower methylation within eosinophils (average 18\% difference in methylation levels in those with

suggests an important role for early life environmental exposures, ${ }^{6,7}$ which can affect epigenetic patterns, leading to changes in gene regulation and, consequently, to altered biological responses contributing to disease susceptibility. ${ }^{89}$ In support of this, epigenetic changes have been observed at specific genomic loci for asthma, including DNA methylation CpG sites, ${ }^{10-15}$ non-coding RNAs, ${ }^{16}$ and microRNA. ${ }^{17}$ Specifically, previous candidate and epigenome-wide studies in peripheral whole blood (or peripheral blood mononuclear cells) have found methylation associations with immune-related genes involved in T-cell regulation and maturation, such as FOXP $3^{18}$ and RUNX3.12 Eosinophil-associated genes have also been implicated, including IL5R $A^{19}$ and IL13; ${ }^{12}$ IL13 is also differentially methylated in airway epithelial cells after stimulation..$^{15}$ Studies on total immunoglobulin E (IgE) levels have also highlighted pathways relevant to eosinophil activation ${ }^{10}$ and lipid or fatty acid metabolism, ${ }^{20}$ with suggested involvement in asthma pathogenesis.

Despite recent progress in asthma epigenetics, several issues-including which DNA methylation sites are robustly associated with asthma across the genome, whether these asthma-associated methylation patterns are already present at birth or develop during childhood, ${ }^{9}$ and whether age-specific patterns can be observed in children from different countries with varying environmental exposures and lifestyles-remain largely unresolved. To answer these questions, we did a large-scale epigenomewide association study (EWAS) of whole-blood DNA in children at preschool age (4-5 years) and school age asthma vs those without), highlighting the importance of the eosinophil as an epigenetic determinant of asthma in childhood. Through clustering of the 14 asthma-associated $\mathrm{CpG}$ sites with whole blood and immune-cell-specific gene expression signatures, we were also able to annotate the asthma-associated $\mathrm{CpG}$ sites to activated immune cell subsets (CD8-positive T cells and natural killer cells).

Implications of all the available evidence

There is now accumulating evidence that methylation status in peripheral blood cells, as well as in disease-relevant tissues such as airway epithelial cells, is altered in patients with asthma and other respiratory diseases. Our study unequivocally shows that reduced whole-blood DNA methylation at specific CpG sites acquired after birth is strongly associated with childhood asthma. These CpG sites and their associated transcriptional profiles indicate activation of eosinophils and cytotoxic $T$ cells. Although we were not able to assess causal links between methylation status and asthma development in our study, it is reasonable to believe that, by targeting methylation status of identified genes (ie, normalising levels), asthma-related disease activity may be regulated. These observations, together with evidence presented before our study, merit further investigation of the role of epigenetics in a clinical context.

(8 years) in four birth cohorts within the Mechanisms of the Development of ALLergy (MeDALL) project ${ }^{21}$, together with a validation and replication study in children (0-16 years) and adults (18-79 years). We subsequently investigated cell-type-specific methylation of the identified CpG sites in eosinophils and respiratory epithelial cells and their related gene-expression signatures.

\section{Methods \\ Study design and participants}

Whole-blood DNA from children from four MeDALL birth cohorts-BAMSE (Barn/Children, Allergy, Milieu, Stockholm, Epidemiology; Sweden), ${ }^{22}$ EDEN (Etude des Determinants pre et post natals du developpement et de la sante de l'Enfant; France), ${ }^{23}$ INMA (Infancia y Medio Ambiente-Sabadel [SAB]; Spain), ${ }^{24}$ and PIAMA (Prevention and Incidence of Asthma and Mite Allergy; the Netherlands) ${ }^{25}$-was investigated in the discovery analysis. $^{26}$

All blood DNA samples from children not participating in the discovery phase were selected for the replication phase from seven MeDALL birth cohorts: BIB (UK), ECA (Norway), Karelia (Finland), PIAMA, RHEA (Greece), ROBBIC (Italy; subsets Bologna and Rome), and INMA (Spain; subsets Gipuzkoa [GIP], Menorca [MEN] and Valencia [VAL]; appendix pp 18-19, 306-07). All of these cohorts, apart from Karelia, were included in the main meta-analysis.

We defined asthma as described previously in the MeDALL study, ${ }^{26}$ requiring two of three criteria present: 
doctor diagnosis of asthma ever; use of asthma medication in the past 12 months; or wheezing or breathing difficulties in the past 12 months.

Medical Ethics Committees of all institutions approved this study. Written informed consent was obtained from parents or legal guardians of all participating children. A full description of the methods is provided in the appendix.

\section{Procedures}

Given the strong effects of age on whole-blood methylation patterns in childhood, ${ }^{9}$ we considered the possibility of age-specific methylation in asthma and performed a discovery analysis at age $4-5$ years and age 8 years. We examined epigenome-wide DNA methylation using Illumina Infinium Human Methylation 450 BeadChips (450K) in 207 children with asthma and 610 controls at age 4-5 years, and 185 children with asthma and 546 controls at age 8 years using a cross-sectional case-control design (figure 1).

We used an independent technology, iPlex (Agena Biosciences), to validate and replicate the selected $\mathrm{CpG}$ sites after quality control of the assays. The most significant $\mathrm{CpG}$ sites at both age $4-5$ years and 8 years were selected for replication (appendix pp 20-22). Next we investigated whether replicated $\mathrm{CpG}$ sites in cord blood predict later asthma in 1316 children participating in BIB, EDEN, INMA-VAL, and ROBBIC (appendix p 23).

We studied cell-type specificity of the asthma association of the replicated $\mathrm{CpG}$ sites in 455 respiratory epithelial cell samples, collected by nasal brushing of 455 16-year-old children, as well as in DNA isolated from blood eosinophils (16 cases with asthma, eight controls [age 2-56 years]) and compared this with whole-blood DNA samples of 167 individuals (age 1-79 years) from families from the Saguenay-Lac-Saint-Jean (SLSJ) region in Canada (appendix p 23). ${ }^{27}$

The association of $\mathrm{CpG}$ methylation with single nucleotide polymorphisms (SNPs) was studied in 496 children, and with matched regional whole-blood gene-expression in 119 children (age 4 years, INMA), and 260 children (age 16 years, BAMSE) using Affymetrix Human Transcriptome Array 2.0 Genechips. ${ }^{28}$ Association between $\mathrm{CpG}$ methylation and whole-genome expression by RNA-seq was assessed in the BIOS consortium dataset. ${ }^{29}$ CpG sites were annotated by GREAT (Genomic Regions Enrichment of Annotations Tool) version 2.0.2. ${ }^{30}$ Functional enrichment analysis was done by overlapping our replicated CpG sites with histone marks and chromatin states of 27 blood cell types in the Roadmap epigenome project. ${ }^{31}$ Gene expression sets associated with replicated CpG sites were interrogated for cell-type specificity using the 500 FG dataset. ${ }^{32}$

\section{Statistical analyses}

Asthma-associated differentially methylated CpG sites were identified by fitting a robust linear regression

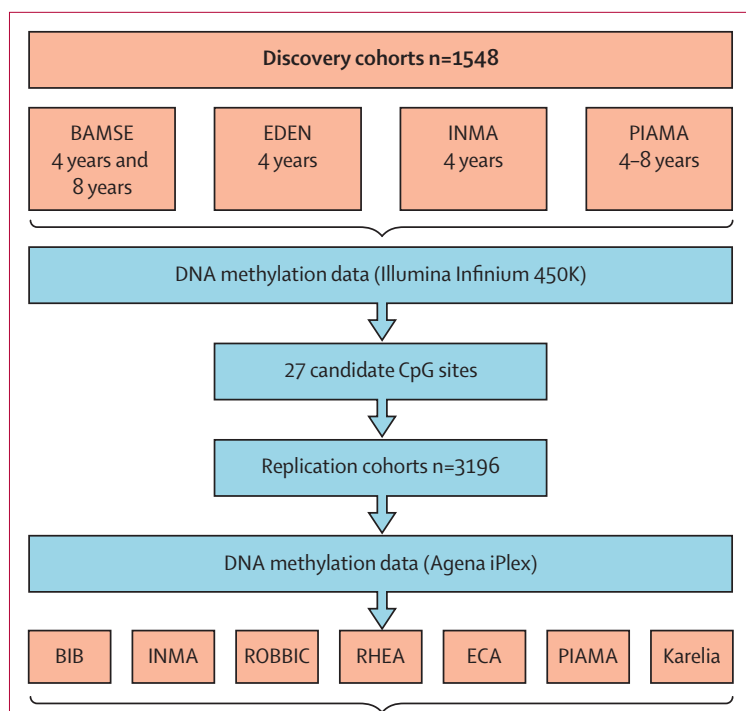

14 replicated CpG sites

Cord blood methylation predicting early childhood asthma (EDEN [n=146], INMA [n=496,] BIB [n=296], ROBBIC [ $n=378]$ )

Extra replication (nasel epithelial [ $n=455]$, eosinophils [ $n=24]$, whole blood with full age range $[n=167,1-79$ years $]$ )

Functional genomics and gene expression (BAMSE [ $n=260]$, INMA [ $n=119]$, $\operatorname{BIOS}[n=2367])$

Gene set and functional enrichment analyses

Genetic association and causality (blood, $n=496$ )

Figure 1: Study design

The EWAS study consisted of a discovery and replication phase, followed by a meta-analysis. The discovery phase was a case-control design with epigenomewide DNA methylation in whole-blood DNA measured by Illumina 450K. The top significant $\mathrm{CpG}$ sites from the discovery phase were selected for replication using the iPlex design in asthma cases and controls from six additional European cohorts $(n=3196)$. Finally, results from the discovery and replication phases were meta-analysed.

method corrected for sex, cohort, and technical covariables. We used models that do not correct for blood cell type as the main model for discovery and replication, and report cell-type-corrected models by inclusion of the estimated cell type proportions as covariates in the sensitivity analysis.

We did inverse variance-weighted fixed-effects meta-analyses with METAL, ${ }^{33}$ or random-effects metaanalysis using Metafor ${ }^{34}$ package with statistical software environment $\mathrm{R}$ in case of a heterogeneity $\chi^{2}$ test $p$ value less than 0.05. Our replicated CpG sites were those that were significantly associated in the meta-analysis of replication samples (Bonferroni correction, $\mathrm{p}<0 \cdot 0019,27$ tests) and passed epigenome-wide significance using Bonferroni correction $\left(\mathrm{p}<1 \cdot 14 \times 10^{-7}, 439306\right.$ tests) after metaanalysis of all studies.
I-Universitat de València,

Valencia, Spain (S Llop, ( Iñiguez); ib-salut, Area de Salut de Menorca, Menorca, Spain (M Torrent PhD) Department of Epidemiology Lazio Regional Health Service, Rome, Italy (F Forastiere PhD, D Porta MSc); Department of Biomedical and Neuromotor sciences, University of Bologna, Bologna, Italy (Prof M P Fantini MD, D Gori MD); Department of Paediatrics, Oslo University Hospital, Oslo, Norway

(Prof K C Lødrup Carlsen MD

$\checkmark$ Hovland PhD,

P Mowinckel MSc,

Prof K-H Carlsen MD); Department of Paediatric and

Adolescent Medicine, University of Oslo, Oslo, Norway

(Prof K C Lødrup Carlsen, Prof K-H Carlsen); Skin and Allergy Hospital, Helsinki University Hospital (Prof T Haahtela MD) and Department of Bacteriology and Immunology, Medicum (H Alenius, N Fyhrquist) University of Helsinki, Helsinki, Finland; Department of Human Genetics, McGill University and Genome Quebec, Innovation Centre Montréal, QC, Canada (A Morin PhD); European Institute for Systems Biology and Medicine, Campus Charle Mérieux - Université de Lyon, CIRI CNRS UMR5308, CNRS-ENS-UCBL-ENS, Lyon, France (S Ballereau PhD N Lemonnier PhD, J Pellet MSc, ( Auffray PhD); Cancer Research UK Cambridge Institute, University of Cambridge, Cambridge, UK (S Ballereau) Department of Pediatrics, Erasmus MC - Sophia Children's Hospital University Medical Center, Rotterdam, The Netherlands (J C de Jongste MD); Department of Epidemiology and Public Health, Swiss Tropical and Public Health Institute, Basel, Switzerland (A Kumar); University of Basel, Basel, Switzerland (A Kumar); Asthma and Airway Disease Research Center, University of Arizona, Tucson, AZ, USA (Prof S Guerra); Faculty of Medicine and Life Sciences and Institute of Biosciences and Medical Technology (BioMediTech), University of Tampere, Tampere, Finland 
(D Greco PhD); Institute for Advanced Biosciences, UGA-INSERM U1209-CNRS UMR5309, Site Santé, Allée des Alpes, La Tronche, France (N Lemonnier); Research BV, Metslawier, the Netherlands (P van der Vlies); National Heart and Lung Institute, Imperial College London, London, UK (Prof M F Moffatt PhD,

ProfW O ( M Cookson PhD);

Department of Internal

Medicine and Radboud Center for Infectious Diseases, Radboud University Medical Center, Nijmegen, the Netherlands (Prof M G Netea PhD); University Hospital, Montpellier, France (Prof J Bousquet MD);

Department of Dermatology,

Charité, Berlin, Germany,

(Prof J Bousquet); National Institute for Health and Welfare, Helsinki, Finland (ProfT Laatikainen PhD); Institute of Public Health and Clinical Nutrition, University of Eastern Finland, Kuopio, Finland (ProfT Laatikainen); Université du Québec à Chicoutimi, Département des sciences fondamentales, Saguenay, QC, Canada (Prof C Laprise PhD): Centre intégré universitaire de santé et de services sociaux du

Saguenay-Lac-Saint-Jean,

305 Saint-Vallier, Saguenay, QC, Canada (Prof C Laprise); Department of Genetics, Physical Anthropology and Animal Physiology, Biocruces Health Research Institute, CIBERDEM, University of the Basque Country UPV-EHU, Leioa-Bizkaia, Spain (J R Bilbao PhD); Department of Medical and Molecular Genetics, King's College London, London, UK (ProfJ Kere); IMIM Hospital del Mar Medical Research Institute, Barcelona, Spain (Prof) Sunyer, Prof J M Anto); Sachs Children's Hospital, Stockholm, Sweden (E Melén); and Centre for Occupational and

Environmental Medicine,

Stockholm County Council,

Stockholm, Sweden (E Melén)

Correspondence to: Prof G H Koppelman, PO Box 30.001, Department of Pediatric Pulmonology and Pediatric

Allergology, Beatrix Children's Hospital, University Medical Center Groningen, CA43, 9700 RB Groningen, the Netherlands g.h.koppelman@umcg.nl
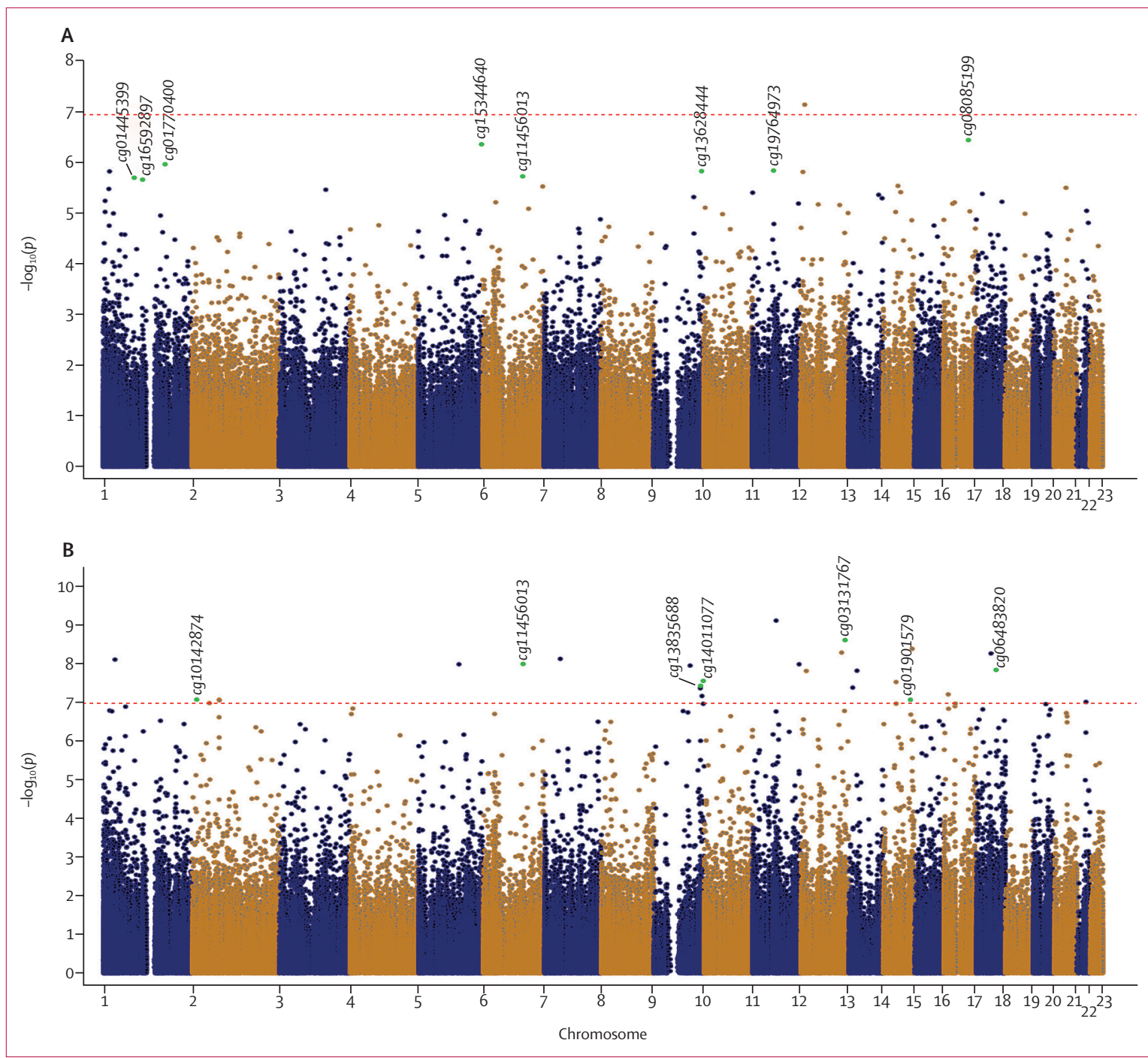

Figure 2: Manhattan plots from the epigenome-wide association studies performed in four European birth cohorts of childhood asthma (discovery) $439306 \mathrm{CpG}$ sites were tested for association with childhood asthma. The red dotted horizontal line represents the Bonferroni-corrected threshold $\left(\mathrm{p}<1 \cdot 14 \times 10^{-7}\right)$ of genome-wide significance. All 14 replicated $\mathrm{CpG}$ sites are marked in green dot and annotated with $\mathrm{CpG}$ site name. (A) Results for the EWAS analysis in children aged 4-5 years $(n=817)$ from the four European cohorts BAMSE, EDEN, INMA and PIAMA. (B) Results for the EWAS meta-analysis in children aged 8 years ( $n=731$ ) from BAMSE and PIAMA.

\section{Role of the funding source}

The funders of the study had no role in the design of the study, data gathering, analysis, interpretation, writing of the report, or in the decision to submit the report for publication. The corresponding author had full access to all the data in the study and had final responsibility for the decision to submit for publication.

\section{Results}

At age 4-5 years, we identified one genome-wide significant CpG site (cg07019303) annotated to ETV6 and $B C L 2 L 14$ ( $\mathrm{p}=7.96 \times 10^{-8}$; appendix pp 20, 308). Therefore, we used a looser threshold (false discovery rate [FDR]adjusted $\mathrm{p}<0 \cdot 10)$ to select $11 \mathrm{CpG}$ sites for replication (appendix p 20) as we considered age-specific effects on asthma. We identified $26 \mathrm{CpG}$ sites that passed the genome-wide significance threshold $\left(\mathrm{p}<1 \cdot 14 \times 10^{-7}\right)$ at age 8 years (figure $2 \mathrm{~B}$, appendix pp 21-22, 308), with the strongest association $\left(\mathrm{p}=8.82 \times 10^{-10}\right)$ found for $\operatorname{cg} 03695871$ in AP5B1, a gene associated with eczema. ${ }^{35}$ Cg11456013 and cg1362844 were identified at both ages $4-5$ years and 8 years, and $35 \mathrm{CpG}$ sites were selected for replication.

IPlex assays for 27 out of the $35 \mathrm{CpG}$ sites selected were available after quality control. 3196 samples from six birth cohorts were selected for our main meta-analysis. 14 of the $27 \mathrm{CpG}$ sites were significantly associated with asthma across childhood after meta-analysis of the six MeDALL replication cohorts and passed the genome-wide significance threshold in the meta-analysis of replication and 


\begin{tabular}{|c|c|c|c|c|c|c|c|c|c|c|c|c|}
\hline & Age group & Chromosome & $\begin{array}{l}\text { Base pair } \\
\text { position* }^{*}\end{array}$ & Gene name $\dagger$ & $\begin{array}{l}\text { Discovery } \\
\text { coefficient }\end{array}$ & $\begin{array}{l}\text { Discovery } \\
\text { p value }\end{array}$ & $\begin{array}{l}\text { Eosinophils- } \\
\text { corrected } \\
\text { p value } \neq\end{array}$ & $\begin{array}{l}\text { Houseman } \\
\text { corrected } \\
\text { p value } \$\end{array}$ & $\begin{array}{l}\text { Replication } \\
\text { coefficient }\end{array}$ & $\begin{array}{l}\text { Replication } \\
\text { p value }\end{array}$ & $\begin{array}{l}\text { Meta- } \\
\text { analysis } \\
\text { coefficient }\end{array}$ & $\begin{array}{l}\text { Meta- } \\
\text { analysis } \\
\text { p value }\end{array}$ \\
\hline cg01445399 & $4-5$ years & 1 & 87596934 & LOC339524 & -0.0120 & $2.15 \times 10^{-6}$ & $4.49 \times 10^{-3}$ & $3.30 \times 10^{-5}$ & -0.0156 & $3.01 \times 10^{-5}$ & -0.0129 & $4.01 \times 10^{-10}$ \\
\hline cg01770400 & $4-5$ years & 1 & 173886485 & SERPINC1 & -0.0080 & $1.17 \times 10^{-6}$ & $2.52 \times 10^{-4}$ & $2.92 \times 10^{-6}$ & -0.0094 & $1.70 \times 10^{-8}$ & -0.0087 & $1.15 \times 10^{-13}$ \\
\hline cg16592897 & $4-5$ years & 1 & 111023201 & PROK1, KCNA10 & -0.0070 & $2.35 \times 10^{-6}$ & $9.91 \times 10^{-4}$ & $6.75 \times 10^{-6}$ & -0.0053 & $2.37 \times 10^{-4}$ & -0.0062 & $3.68 \times 10^{-9}$ \\
\hline $\operatorname{cg} 15344640$ & $4-5$ years & 5 & 176774729 & MXD3, LMAN2 & -0.0090 & $4.77 \times 10^{-7}$ & $7.74 \times 10^{-4}$ & $1.04 \times 10^{-5}$ & -0.0105 & $1.93 \times 10^{-4}$ & -0.0095 & $4.09 \times 10^{-10}$ \\
\hline cg11456013 & $4-5$ years & 6 & 111192760 & AMD1 & -0.0130 & $2.03 \times 10^{-6}$ & $1.23 \times 10^{-2}$ & $4.93 \times 10^{-5}$ & -0.0141 & $3.37 \times 10^{-11}$ & -0.0135 & $3.79 \times 10^{-16}$ \\
\hline $\operatorname{cg} 13628444$ & $4-5$ years & 9 & 134883788 & $\begin{array}{l}\text { RAPGEF1, } \\
\text { MED27 }\end{array}$ & -0.0110 & $1.60 \times 10^{-6}$ & $2.96 \times 10^{-3}$ & $4.40 \times 10^{-5}$ & -0.0115 & $5.71 \times 10^{-5}$ & -0.0111 & $3.85 \times 10^{-10}$ \\
\hline cg19764973 & $4-5$ years & 11 & 59525433 & STX3, MRPL16 & -0.0090 & $1.56 \times 10^{-6}$ & $8.70 \times 10^{-4}$ & $1.74 \times 10^{-5}$ & -0.0136 & $5.16 \times 10^{-10}$ & -0.0112 & $1.14 \times 10^{-14}$ \\
\hline cg08085199 & $4-5$ years & 16 & 69170548 & CHTF8, CIRH1A & -0.0120 & $3.94 \times 10^{-7}$ & $1.23 \times 10^{-3}$ & $2.85 \times 10^{-5}$ & -0.0111 & $2.84 \times 10^{-7}$ & -0.0117 & $5.79 \times 10^{-13}$ \\
\hline cg10142874 & 8 years & 2 & 11917623 & TRIB2, LPIN1 & -0.0158 & $9.42 \times 10^{-8}$ & $9.89 \times 10^{-4}$ & $2.05 \times 10^{-6}$ & -0.0181 & $1.64 \times 10^{-6}$ & -0.0167 & $1.20 \times 10^{-12}$ \\
\hline cg11456013 & 8 years & 6 & 111192760 & AMD1 & -0.0181 & $1.15 \times 10^{-8}$ & $3.28 \times 10^{-4}$ & $9.67 \times 10^{-7}$ & -0.0124 & $1.81 \times 10^{-7}$ & -0.0144 & $4.00 \times 10^{-14}$ \\
\hline cg14011077 & 8 years & 9 & 138362327 & $\begin{array}{l}\text { PPP1R26, } \\
\text { OLFM1 }\end{array}$ & -0.0154 & $3.12 \times 10^{-8}$ & $8.60 \times 10^{-4}$ & $2.76 \times 10^{-7}$ & -0.0138 & $6.02 \times 10^{-5}$ & -0.0148 & $1.06 \times 10^{-11}$ \\
\hline cg13835688 & 8 years & 9 & 130859454 & $\begin{array}{l}\text { SLC25A25, } \\
\text { PTGES2 }\end{array}$ & -0.0212 & $4.18 \times 10^{-8}$ & $4.41 \times 10^{-6}$ & $5.64 \times 10^{-8}$ & -0.0193 & $3.95 \times 10^{-4}$ & -0.0205 & $9.01 \times 10^{-11}$ \\
\hline cg03131767 & 8 years & 12 & 123446272 & VPS37B, ABCB9 & -0.0159 & $2.79 \times 10^{-9}$ & $2.51 \times 10^{-4}$ & $2.49 \times 10^{-7}$ & -0.0134 & $2.08 \times 10^{-5}$ & -0.0148 & $4.48 \times 10^{-13}$ \\
\hline cg01901579 & 8 years & 14 & 95615731 & DICER1, CLMN & -0.0177 & $9.52 \times 10^{-8}$ & $5.64 \times 10^{-5}$ & $1.03 \times 10^{-6}$ & -0.0188 & $5.58 \times 10^{-11}$ & -0.0183 & $2.55 \times 10^{-17}$ \\
\hline $\operatorname{cg} 06483820$ & 8 years & 17 & 55167149 & $M S I 2, A K A P 1$ & -0.0159 & $1.63 \times 10^{-8}$ & $2.65 \times 10^{-4}$ & $1.27 \times 10^{-6}$ & -0.0119 & $2.20 \times 10^{-5}$ & -0.0139 & $2.30 \times 10^{-12}$ \\
\hline \multicolumn{13}{|c|}{$\begin{array}{l}\text { *Base pair position according to Genome build 37. The CpG sites were annotated by GREAT version 2.0.2. FEosinophils-corrected p value: p value from discovery study after correct estimated cell counts, } \\
\text { including eosinophils (CD8 and CD4T cells, natural killer cells, B cells, monocytes, eosinophils, neutrophils). SHouseman corrected p value: } p \text { value from discovery study after correct estimated cell counts with } \\
\text { default Houseman setting (CD8 and CD4T cells, natural killer cells, B cells, monocytes, granulocytes). } 9 \text { The same CpG sites were detected in both age groups. }\end{array}$} \\
\hline
\end{tabular}

discovery cohorts (table 1, figure 2A, appendix pp 24-29). The expression and function of genes annotated to replicated $\mathrm{CpG}$ sites are described in the appendix (pp 30-35), as are the genomic annotations of all CpG sites and regional co-methylation plots (appendix pp 309-30). The most significant association $\left(\mathrm{p}=2 \cdot 55 \times 10^{-17}\right)$ was observed for cg01901579 in DICER1 (appendix p 331). Specific replication at age 4 years is provided in the appendix ( $p$ 36), but could not be done at age 8 years due to low power. Stratification of asthma cases at age 4 years by the presence of specific IgE levels to aeroallergens showed association of both specific IgE-positive and IgE-negative asthma with the top $11 \mathrm{CpG}$ sites (from the 4-year discovery analyses), with, on average, stronger results for specific IgE-positive asthma (appendix pp 37-38). Methylation levels in patients with asthma were consistently lower than those in controls for all 14 replicated CpG sites, with small effects in whole blood in the discovery cohorts (appendix pp 332-35). To evaluate potential SNP effects within the probe for the 14 replicated $\mathrm{CpG}$ sites, the $\beta$ value distributions were visually assessed in the discovery cohorts (appendix p 336), and no bimodal distribution was detected that could reveal an underlying SNP effect within the probe. Additionally, the residuals were normally distributed. Homogeneity testing showed consistent effects across childhood (4-16 years) for all replicated sites $\left(\mathrm{p}_{\text {heterogeneity }}>0.05 ; \quad I^{2}<25 \%\right.$; appendix pp 337-365). Strikingly, all 14 CpG sites were significantly associated with asthma in a second replication study using whole-blood DNA from Canadian families (age 1-79 years; table 2). However, methylation of the 14 asthma-associated CpG sites in 1316 cord-blood DNA samples did not significantly predict asthma development at age 3-4 years in four different cohorts (appendix p 39).

In our discovery samples, white blood cell composition was measured in the INMA cohort (appendix p 40) and estimated in all four cohorts (appendix p 40). Asthma was significantly associated with lower estimated CD4-positive and CD8-positive T-cell subsets in whole blood, but not with blood eosinophil counts. However, the association of CpG methylation with asthma became less significant after eosinophil and neutrophil correction ( $p$ values after correction ranged from $1.23 \times 10^{-2}$ to $4.41 \times 10^{-6}$ vs $1 \cdot 17 \times 10^{-6}$ to $2 \cdot 79 \times 10^{-9}$ before correction; table 1 , appendix pp 24-29). Using Reinius' blood-cell-type specific methylation data, ${ }^{36}$ we found that 11 of the 14 asthmaassociated $\mathrm{CpG}$ sites had relatively lower methylation levels in eosinophils compared with other blood cell types $\left(\mathrm{p}=1 \cdot 92 \times 10^{-24}\right.$, Fisher's exact test; appendix pp 41-42).

In the SLSJ cohort, we found all $14 \mathrm{CpG}$ sites within eosinophils to be significantly associated with asthma in individuals with a very broad age range of 2-56 years (appendix p 23). CpG methylation in eosinophils was on average $18 \%$ lower in participants with asthma (table 2, appendix pp 366-79). Furthermore, we found significant differential methylation in relation to asthma for five of $14 \mathrm{CpG}$ sites in nasal respiratory epithelial cells ${ }^{13}$ from children aged 16 years $\left(\mathrm{p}_{\text {enrichment }}=3 \cdot 8 \times 10^{-4}\right.$; table 2$)$.

Four of $14 \mathrm{CpG}$ sites were associated with SNPs within $250 \mathrm{~kb}$ (cis-MeQTL; appendix p 43), including
For more on GREAT (Genomic Regions of Annotations Tool see http://bejerano.stanford. edu/great/

See Online for appendix 


\begin{tabular}{|c|c|c|c|c|c|c|c|}
\hline & Gene name & $\begin{array}{l}\text { Whole blood } \\
\text { coefficient* }\end{array}$ & $\begin{array}{l}\text { Whole blood } \\
\text { p value } †\end{array}$ & $\begin{array}{l}\text { Eosinophils } \\
\text { coefficient } \neq\end{array}$ & $\begin{array}{l}\text { Eosinophils } \\
\text { p value } \$\end{array}$ & $\begin{array}{l}\text { Nasal epithelial } \\
\text { coefficient } \Upsilon\end{array}$ & $\begin{array}{l}\text { Nasal epithelial } \\
\text { p value|l }\end{array}$ \\
\hline cg01901579 & DICER1, CLMN & -0.0188 & $1.44 \times 10^{-6}$ & $-0 \cdot 180$ & 0.0030 & -0.0112 & 0.0008 \\
\hline cg13628444 & RAPGEF1, MED27 & -0.0152 & $5.08 \times 10^{-5}$ & -0.225 & 0.0009 & -0.0159 & 0.0037 \\
\hline cg19764973 & STX3, MRPL16 & -0.0072 & 0.0001 & -0.264 & 0.0228 & -0.0113 & 0.0052 \\
\hline cg01445399 & LOC339524 & -0.0153 & 0.0005 & -0.212 & 0.0191 & -0.0124 & 0.0148 \\
\hline $\operatorname{cg} 13835688$ & SLC25A25, PTGES2 & -0.0159 & $7.94 \times 10^{-5}$ & -0.216 & 0.0004 & $-0 \cdot 0120$ & 0.0190 \\
\hline $\operatorname{cg} 11456013$ & AMD1 & -0.0147 & 0.0004 & -0.189 & 0.0023 & -0.0069 & 0.2074 \\
\hline $\operatorname{cg} 15344640$ & MXD3, LMAN2 & -0.0089 & 0.0009 & -0.236 & 0.0038 & -0.0035 & 0.3413 \\
\hline cg01770400 & SERPINC1 & -0.0050 & 0.0111 & $-0 \cdot 140$ & 0.0017 & -0.0032 & 0.3610 \\
\hline cg06483820 & MSI2, AKAP1 & -0.0107 & 0.0060 & -0.185 & 0.0064 & 0.0046 & 0.4942 \\
\hline cg03131767 & VPS37B, ABCB9 & -0.0106 & 0.0117 & $-0 \cdot 215$ & 0.0037 & -0.0045 & 0.6359 \\
\hline cg10142874 & TRIB2, LPIN1 & -0.0201 & $1.16 \times 10^{-5}$ & -0.191 & 0.0006 & -0.0019 & 0.6745 \\
\hline cg08085199 & CHTF8, CIRH1A & -0.0148 & 0.0014 & -0.229 & 0.0092 & 0.0027 & 0.7121 \\
\hline cg16592897 & PROK1, KCNA10 & -0.0066 & 0.0223 & -0.070 & 0.0021 & -0.0004 & 0.9072 \\
\hline cg14011077 & PPP1R26, OLFM1 & $-0 \cdot 0123$ & 0.0205 & -0.108 & 0.0050 & -0.0010 & 0.9099 \\
\hline \multicolumn{8}{|c|}{$\begin{array}{l}\text { *Regression coefficient from association study of SLSJ whole blood. } \dagger p \text { value from association study of SLSJ whole blood. } ¥ \text { Regression coefficient from association study of } \\
\text { SLSJ isolated eosinophils. Sp value from association study of SLSJ isolated eosinophils. IRegression coefficient from association study of PIAMA nasal epithelial cells. IIp value } \\
\text { from association study of PIAMA nasal epithelial cells. }\end{array}$} \\
\hline
\end{tabular}

rs9425436, which was associated with $\mathrm{CpG}$ methylation of $\operatorname{cg} 01770400$ in SERPINC1 $\left(\mathrm{p}=5 \cdot 6 \times 10^{-4}\right)$. rs 9425436 was in strong linkage disequilibrium $\left(\mathrm{r}^{2}=0 \cdot 95\right)$ with $r s 4652298$ in ZBTB37, which has previously been shown to be associated with asthma $\left(\mathrm{p}=2 \cdot 7 \times 10^{-3}\right)$ in the GABRIEL GWAS. ${ }^{4}$ Mediation analysis revealed that $14.4 \%$ of the SNP rs9425436 effect on asthma was due to CpG methylation of cg01770400 (appendix p 43). 13 of 14 replicated $\mathrm{CpG}$ sites were significantly enriched for enhancer markers in whole-blood Roadmap and Encode data $^{31}$ (appendix pp 44-45).

Four asthma-associated $\mathrm{CpG}$ sites were significantly associated with gene expression in cis in BAMSE (appendix p 46), but this was not replicated in the smaller INMA dataset (appendix $p$ 47). In the larger BIOS dataset, ${ }^{29}$ which contains participants from the Dutch LifeLines Deep study, ${ }^{37}$ Leiden Longevity Study, ${ }^{38}$ and the Netherlands Twin register, ${ }^{39}$ we analysed the association of the $14 \mathrm{CpG}$ sites with gene expression in cis in 2367 adults, and identified ten of the 14 asthma-associated CpG sites to be associated with 22 gene transcripts (appendix p 48), with two CpG-gene transcript pairs (cg06483830-SCPEP1 and cg14011077-PPP1R26) also observed in the BAMSE dataset (appendix p 46).

All CpG sites showed significant associations with whole-blood gene expression (mean number of associated transcripts 351 [SD 228]). All associated transcripts are shown per CpG site in the appendix (pp 49-238). We identified three distinct gene expression patterns in whole blood by hierarchical clustering. The first cluster was inversely associated with methylation levels of seven $C p G$ sites, and included genes such as SLC29A1, SIGLEC8, IL5RA, and ADORA3 (figure 3A, appendix pp 49-238). A second cluster was strongly associated with cg16592897 and six other CpG sites, and included genes such as CCR7, CHMP7, and LEF1. After correction for eosinophil blood counts, association of the first cluster was attenuated, confirming this as an eosinophilic gene signature, whereas the association of the second cluster became more prominent. In contrast, the third cluster, containing TBX21, EOMES, CCL5, and $G Z M B$, showed negative correlation with four asthma CpG methylation levels (figure 3B, appendix pp 239-305). An RNA-seq dataset of peripheral blood mononuclear cells fluorescence-activated cell-sorted into highly defined leucocyte subsets ${ }^{32}$ identified the second cluster as a naive CD4 and CD8 T cell gene signature that was decreased, and the third cluster as an effector and memory CD8 T cell and natural killer cell profile that was increased with reduced $\mathrm{CpG}$ methylation (appendix pp 380-82).

\section{Discussion}

This large consortium-based meta-analysis identified 14 CpG sites in whole blood to be associated with childhood asthma. Consistently lower methylation of these CpG sites was observed in individuals with asthma from age 4-5 years until adolescence. Strongly reduced methylation at asthma-associated $\mathrm{CpG}$ sites within isolated eosinophils clearly indicated that lower whole-blood DNA methylation levels in asthma were due not only to altered eosinophil numbers in whole blood, but also to reduced methylation at those specific $\mathrm{CpG}$ sites in this cell type. Finally, through clustering of the 14 asthma associated CpG sites with whole-blood and immune-cell-specific gene expression signatures, we were able to annotate the asthma-associated 


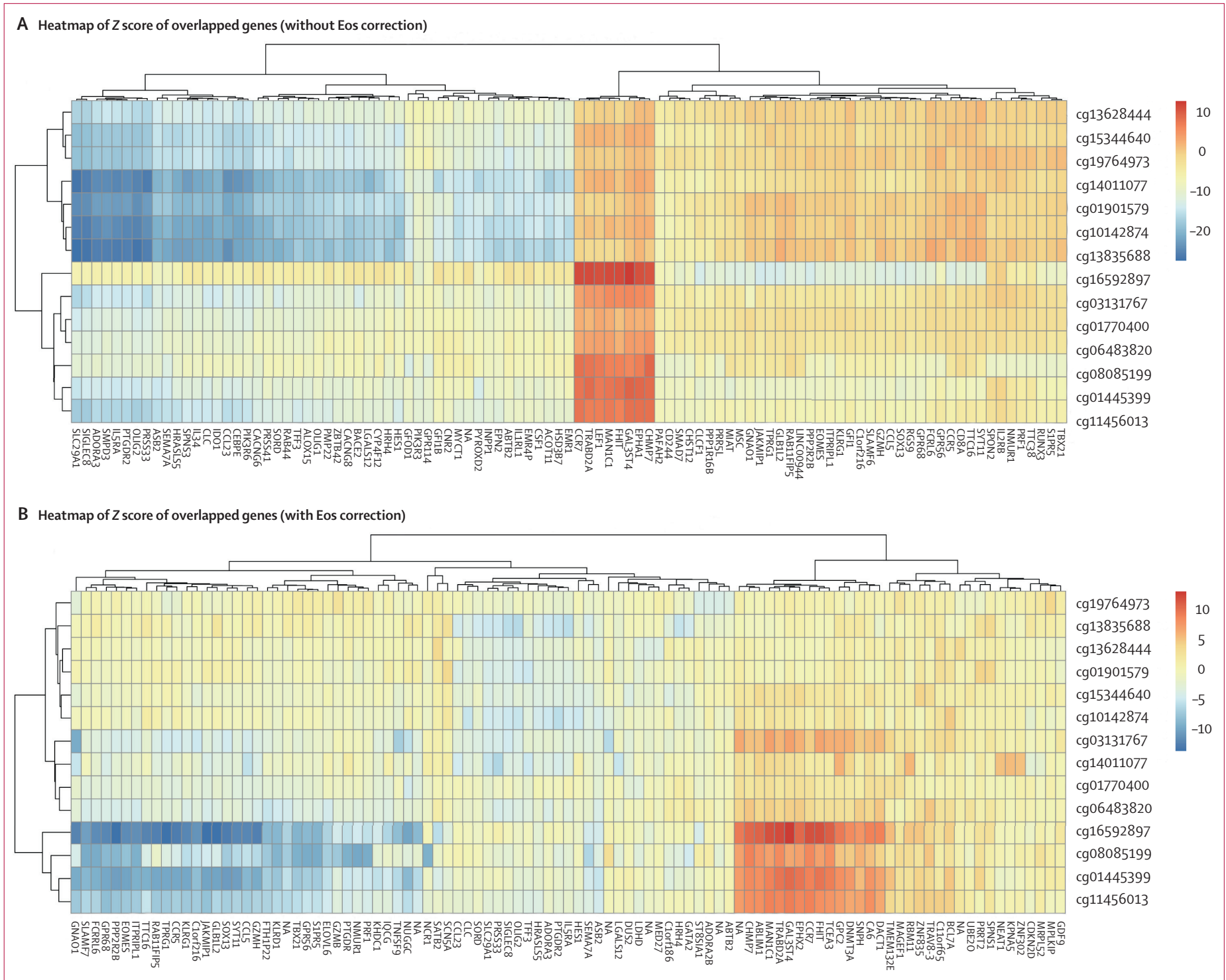

Figure 3: Heatmap and hierarchical clustering of z-scores of the genes most significantly associated with methylation levels of the $14 \mathrm{CpG}$ sites

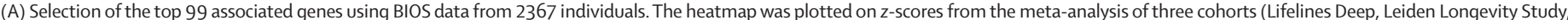

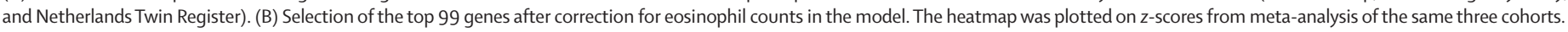

CpG sites to (activated) eosinophils and CD8-positive $\mathrm{T}$ cells and natural killer cells.

DNA methylation can be strongly affected by ageing and environmental exposures. ${ }^{8,9}$ We observed remarkably consistent patterns of DNA methylation associated with childhood asthma across different age groups and in participants from different countries. This suggests that our asthma-associated CpG sites are consistent findings in childhood asthma from the age of 4 years onwards, but we did not find this association at birth, indicating postnatal effects on methylation of the $14 \mathrm{CpG}$ sites identified in our study. Recently, cord-blood methylation of a CpG site in another asthma gene, SMAD3, was found to be associated with childhood asthma in children of mothers with asthma. ${ }^{11}$ Maternal smoking during pregnancy, a risk factor for childhood asthma, showed strong effects on methylation of $6073 \mathrm{CpG}$ sites in DNA isolated from cord blood. ${ }^{8}$ One of these CpG sites was associated with asthma in our study (LMAN2), but we did not identify a mediating effect of smoking on asthma through this $\mathrm{CpG}$ (data not shown). Thus, asthma-associated DNA methylation patterns identified in this study are likely to be the result of postnatal environmental influences, pathophysiological processes related to asthma, or both. We performed detailed functional analysis of the asthma-associated $\mathrm{CpG}$ sites to start identifying these processes.

Part of the reduced DNA methylation of the $14 \mathrm{CpG}$ sites in asthma was explained by eosinophil numbers, 
since the significance of the association was reduced after correction for predicted eosinophil percentage in peripheral blood. $^{20}$ Importantly, strongly reduced methylation of the $\mathrm{CpG}$ sites in purified eosinophils retained an association with asthma, highlighting that eosinophils are epigenetically altered in asthma. We observed a larger effect size in purified eosinophils (on average $18 \%$ lower in individuals with asthma than in those without asthma) compared with whole blood (on average $1-2 \%$ lower in individuals with asthma than in those without asthma), consistent with a functional effect on gene expression in eosinophils. As predicted, eosinophil counts were not significantly different between patients with asthma and controls in our discovery cohorts, suggesting that our results reflect the presence of a different subset or activation state of eosinophils, rather than a change in eosinophil counts per se. Although eosinophils are linked to subphenotypes of asthma such as the $\mathrm{T}$ helper 2-type subset, ${ }^{40}$ our study provides unequivocal data supporting their involvement at the epigenetic level in mild-tomoderate asthma in children. This adds to the genetic findings ${ }^{41}$ that SNPs regulating blood eosinophil counts significantly overlap with asthma GWAS loci, such as IL5, IL33, IL1RL1, and TSLP. Functional interpretation using Roadmap epigenomics data revealed that asthmaassociated $\mathrm{CpG}$ sites were significantly enriched for enhancer markers, again suggesting a cell-type-specific regulatory role in gene expression. ${ }^{42}$

Correlation of our $14 \mathrm{CpG}$ sites with genome-wide gene expression revealed three clusters of associated genes. Because all replicated $14 \mathrm{CpG}$ sites show consistently reduced DNA methylation in patients with asthma, the cluster encompassing known eosinophil signature genes $^{43}$ that are negatively associated with methylation levels of asthma-associated $\mathrm{CpG}$ sites is therefore predicted to show increased expression in asthma. A second cluster with increased predicted expression in asthma is characterised by effector and memory CD8 $\mathrm{T}$ cell and natural killer cell genes. A third cluster with reduced predicted expression in asthma was identified as naive CD4 and CD8 T cells. These data indicate that whole-blood DNA from children with asthma carries CpG methylation marks associated with reduced activity of naive $\mathrm{T}$ cells and increased activity of effector and memory CD8 $\mathrm{T}$ cells and natural killer cells.. ${ }^{4}$ This asthma-associated shift in cellular activity might be due to different environmental factors such as traffic-related air pollution or infectious agents, including intracellular bacteria or viruses. ${ }^{45}$ Further studies are needed in well characterised populations of children with asthma to address cell-type-specific methylation signatures together with RNA-seq data in effector memory CD8-positive $\mathrm{T}$ cells and natural killer cells to disentangle a cell proportion effect from differential cell activation in asthma. Thus, asthma-associated whole-blood methylation patterns represent an epigenetic fingerprint of (activated) immune cell subsets acquired in childhood. Given that large methylation differences were seen in eosinophils from individuals with asthma compared with those without asthma, we believe that our findings could have a direct clinical impact in the future by aiding diagnostics, guiding drug discovery programmes, or both. For example, rapid tests for eosinophil methylation status available for clinical use could be a valuable diagnostic tool to identify children with asthma, and we encourage such new research efforts. Although we are not able to assess causal links between methylation status and asthma development in our study, it is reasonable to believe that, by targeting the methylation status of identified genes (ie, normalising levels), asthmarelated disease activity might be regulated.

Comparing the $14 \mathrm{CpG}$ sites with a DNA methylation study of asthma in US inner cities, ${ }^{12}$ we did not identify overlapping $\mathrm{CpG}$ sites. However, three of the genomewide significant CpG sites (cg01770400, cg20503329, cg09447105; appendix pp 20-22) were previously reported in an EWAS for total $\operatorname{IgE},^{10}$ compatible with shared regulation of these $\mathrm{CpG}$ sites in asthma and IgE-related traits. None of these annotated genes was previously implicated in (genetic) studies of asthma (see appendix, pp 30-35, for an overview); however, several of these genes (DICER1, STX3, LIPIN1) have known functions that might be relevant to asthma development. The most significant CpG site was annotated to DICER1 (appendix $\mathrm{p}$ 331). DICER1 is a member of the ribonuclease III family, involved in the generation of microRNAs, which modulate gene expression at the post-transcriptional level. SYNTAXIN3 (STX3) is relevant for epithelial polarity and regulates exocytosis. ${ }^{46}$ Epithelial polarity is important to maintain epithelial barrier function, a process that is impaired in asthma. ${ }^{47}$ STX3 plays an important part in the release of chemokines CXCL8, CCL2, CCL3, and CCL4 by human mast cells. ${ }^{48}$ LIPIN1 is a $\mathrm{Mg}^{2+}$-dependent phosphatidic acid phosphohydrolase, which has been shown to negatively regulate mast cell degranulation and the anaphylactic response through inhibiting the PKC-SNAP-23 pathway. ${ }^{49}$ LIPIN1 also has a role in macrophage proinflammatory activation during Toll-like receptor (TLR) signalling. After TLR4 stimulation, Lipin-1-deficient macrophages showed a decreased production of diacylglycerol and activation of MAP kinases and AP-1, accompanied by a reduced production of proinflammatory cytokines such as IL-6, IL-12, and IL-23, or enzymes such as inducible nitric oxide synthase. ${ }^{50}$

Finally, LMAN2 (lectin, mannose binding 2) encodes a type I transmembrane lectin that shuttles between the endoplasmic reticulum, the Golgi apparatus, and the plasma membrane, and has previously been implicated in sphingolipid transport. ${ }^{5}$

Our study has some strengths and some limitations. To our knowledge, this is the first study to combine EWAS with an extensive validation and replication study of CpG 
sites using an independent laboratory method in more than 5000 children. One limitation of our work is that we used a questionnaire-based approach to define asthma at age 4-5 years based on doctor's diagnosis, symptoms, and use of asthma medication. Although we acknowledge that self-reported asthma at age $4-5$ years could be sensitive to misclassification of transient wheezing, our finding that eight of the $11 \mathrm{CpG}$ sites selected from the age 4-5 years discovery sample were significantly associated with asthma throughout childhood strongly suggests that we found robust asthma $\mathrm{CpG}$ sites. However, we cannot ignore that the challenges of diagnosing asthma in young children might partly explain why we identified only one genome-wide $\mathrm{CpG}$ site in the initial EWAS discovery on 4-5-year olds. Since we a priori considered age-specific effects on DNA methylation in asthma, two sets of CpG sites were selected for replication at age 4-5 years and 8 years. Although different significance cutoffs were used for this initial selection (FDR $<0.1$ at age $4-5$ years, Bonferroni significance at age 8 years), this did not affect the final outcome because all CpG sites passed robust criteria for replication (Bonferroni significance in the replication analysis, as well as in the final combined meta-analysis of discovery and replication studies). Additionally, replication analyses of our 14 top hits in the SLSJ cohort allowed us not only to assess methylation profiles in purified eosinophils, but also to show that hypomethylation of these $\mathrm{CpG}$ sites was observed in participants with asthma at a broader age range (age 1-79 years). A second limitation is that the CpG selection was limited to the $450 \mathrm{~K}$ platform that, by design, only covers $1.6 \%$ of all methylation sites of the genome. We would expect to find many more asthma-associated $\mathrm{CpG}$ sites using whole-methylome sequencing in the future. Third, we can address CpG loci but not $\mathrm{CpG}$ regions in our study since $\mathrm{CpG}$ regions cannot be replicated in the targeted iPlex design. Fourth, small differences in methylation levels between cases and controls were observed in the whole-blood samples, whereas substantial differences were seen in purified eosinophils. This indicates dilution of the strong methylation effects (in eosinophils) if whole blood is used for analyses. Finally, we observed replication of five of the $14 \mathrm{CpG}$ sites in respiratory nasal epithelial cells, but cannot exclude the possibility that (part of) this signal is due to admixture of eosinophils in epithelial brushings.

In conclusion, our study identified consistently reduced DNA methylation levels in $14 \mathrm{CpG}$ sites to be associated with asthma across childhood from ages 4 to 16 years, but not at birth. Asthma-associated whole-blood DNA methylation profiles were strongly driven by lower methylation within eosinophils, highlighting the importance of eosinophils as an epigenetic determinant of asthma. Additionally, CpG methylation patterns identified an early-life shift from naive $\mathrm{T}$ cell populations towards effector and memory CD8 and natural killer cell subsets, indicating a potentially crucial role for host- virus interactions in asthma inception. Our study reveals whole-blood methylation signatures that represent an epigenetic fingerprint of (activated) eosinophil and CD8-positive $\mathrm{T}$ cell and natural killer cell subsets acquired in childhood.

\section{Contributors}

GHK and EM conceived the project and coordinated the analyses. CJX analysed epigenome-wide data for discovery and replication. GHK, EM, CJX, CS and MCN drafted the manuscript. MCN contributed to the biological interpretation. CS, OG, SKM, AK, GP, DG, LR, JK, and EM provided the biological materials and phenotype information of BAMSE. $\mathrm{MBu}, \mathrm{MBa}, \mathrm{SL}, \mathrm{MT}, \mathrm{SG}$, JGA, CI, JRB, and JS provided the biological materials and phenotype information of INMA. NB and IAM provided the biological materials and phenotype information of EDEN. UG, JCdJ, HAS, and $\mathrm{BB}$ provided the biological materials and phenotype information of PIAMA. DM, RRCM, RA, and JW provided the biological materials and phenotype information of BIB. LC and MK provided the biological materials and phenotype information of Rhea. FF, MPF, DG, and DP provided the biological materials and phenotype information of ROBBIC. KCL, VH, PM, and KHC provided the biological materials and phenotype information of ECA. TH, HA, NF, and TL provided the biological materials and phenotype information of Karelia. OG implemented the shadow analysis of EWAS. CL designed, recruited, evaluated, and managed the SLSJ study. AM, MFM, WOCMC, and CL implemented and coordinated replication in SLSJ. MK constructed the MeDALL asthma phenotypes. SKM did eQTM analysis of BAMSE 16 years. CJX did eQTM analysis of BIOS data. MJB contributed Great and Roadmap data annotation. RAG, YL, CW, and MGN implemented and coordinated RNA seq analysis in 500G. CJV did PIAMA RNA seq analysis. SB, NL, JP, and CA implemented and coordinated eQTM analysis in INMA. BvR, SAJ, PvDV, $\mathrm{CCvD}$, and CS designed and produced the 450k and iplex data. JMA and JB coordinated the MeDALL project. All authors were involved in data interpretation, and read and approved the manuscript.

\section{Declaration of interests}

JP reports grants from the European Commission, during the conduct of the study. JB reports personal fees from Almirall, AstraZeneca, Chiesi, GSK, Meda, Menarini, Merck, MSD, Novartis, Sanofi-Aventis, Takeda, Teva, and Uriach, outside of the submitted work. MCN reports grants from EU FP7 program - MeDALL project, during the conduct of the study; and grants from GSK Ltd and personal fees from DC4U, outside of the submitted work. CL reports a pending patent named "Refractory asthmatics with high levels of IgE and low levels of methylation". EM reports grants from the EU (ERC) during conduct of the study, and lecture fees from Thermo Fisher Scientific and Meda outside of the submitted work. GHK reports grants from the EU and grants from Lung Foundation of the Netherlands, during the conduct of the study; and grants from TETRI foundation, grants from UBBO EMMIUS

foundation, and grants from TEVA the Netherlands, outside of the submitted work. All other authors declare no competing interests.

\section{Acknowledgments}

We are grateful to all children and families who participated in this study. We especially thank Professsor Dirkje Postma for helpful comments and support during the course of this study. The Mechanisms of the Development of ALLergy (MeDALL) EU project was supported by the seventh Framework programme (grant agreement number 261357). The Biobank-Based Integrative Omics Studies (BIOS) Consortium is funded by BBMRI-NL, a research infrastructure financed by the Dutch government (NWO 184.021.007). ISGlobal is a member of the CERCA Programme, Generalitat de Catalunya.

\section{References}

1 Eder W, Ege MJ, von Mutius E. The asthma epidemic. $N$ Engl J Med 2006; 355: 2226-35.

2 Martinez FD, Wright AL, Taussig LM, Holberg CJ, Halonen M, Morgan WJ. Asthma and wheezing in the first six years of life. N Engl J Med 1995; 332: 133-38.

3 McGeachie MJ, Stahl EA, Himes BE, et al. Polygenic heritability estimates in pharmacogenetics: focus on asthma and related phenotypes. Pharmacogenet Genomics 2013; 23: 324-28. 
4 Moffatt MF, Gut IG, Demenais F, et al. A large-scale, consortium-based genomewide association study of asthma. N Engl J Med 2010; 363: 1211-21.

5 Ober C, Nicolae DL. Meta-analysis of genome-wide association studies of asthma in ethnically diverse North American populations. Nat Genet 2011; 43: 887-92.

6 Stein MM, Hrusch CL, Gozdz J, et al. Innate immunity and asthma risk in Amish and Hutterite farm children. N Engl J Med 2016; 375: 411-21.

7 Ege MJ, Mayer M, Normand A-C, et al. Exposure to environmental microorganisms and childhood asthma. N Engl J Med 2011; 364: 701-09.

8 Joubert BR, Felix JF, Yousefi P, et al. DNA methylation in newborns and maternal smoking in pregnancy: genome-wide consortium meta-analysis. Am J Hum Genet 2016; 98: 680-96.

9 Xu C-J, Bonder MJ, Söderhäll C, et al. The emerging landscape of dynamic DNA methylation in early childhood. BMC Genomics 2017; 18: 25 .

10 Liang L, Willis-Owen SAG, Laprise C, et al. An epigenome-wide association study of total serum immunoglobulin E concentration. Nature 2015; 520: 670-74.

11 DeVries A, Wlasiuk G, Miller SJ, et al. Epigenome-wide analysis links SMAD3 methylation at birth to asthma in children of asthmatic mothers. J Allergy Clin Immunol 2017; 140: 534-42.

12 Yang IV, Pedersen BS, Liu A, et al. DNA methylation and childhood asthma in the inner city. J Allergy Clin Immunol 2015; 136: 69-80.

13 Yang IV, Richards A, Davidson EJ, et al. The nasal methylome: a key to understanding allergic asthma. Am J Respir Crit Care Med 2017; 195: 829-31.

14 Nicodemus-Johnson J, Myers RA, Sakabe NJ, et al. DNA methylation in lung cells is associated with asthma endotypes and genetic risk. JCI Insight 2016; 1: e90151.

15 Nicodemus-Johnson J, Naughton KA, Sudi J, et al. Genome-wide methylation study identifies an IL-13-induced epigenetic signature in asthmatic airways. Am J Respir Crit Care Med 2016; 193: 376-85.

16 Austin PJ, Tsitsiou E, Boardman C, et al. Transcriptional profiling identifies the long noncoding RNA plasmacytoma variant translocation (PVT1) as a novel regulator of the asthmatic phenotype in human airway smooth muscle. J Allergy Clin Immunol 2017; 139: 780-89.

17 Maes T, Cobos FA, Schleich F, et al. Asthma inflammatory phenotypes show differential microRNA expression in sputum. J Allergy Clin Immunol 2016; 137: 1433-46.

18 Nadeau K, McDonald-Hyman C, Noth EM, et al. Ambient air pollution impairs regulatory T-cell function in asthma. J Allergy Clin Immunol 2010; 126: 845-852.e10.

19 Arathimos R, Suderman M, Sharp GC, et al. Epigenome-wide association study of asthma and wheeze in childhood and adolescence. Clin Epigenetics 2017; 9: 112.

20 Chen W, Wang T, Pino-Yanes M, et al. An epigenome-wide association study of total serum IgE in Hispanic children. J Allergy Clin Immunol 2017; 140: 571-77.

21 Bousquet J, Anto J, Auffray C, et al. MeDALL (Mechanisms of the Development of ALLergy): an integrated approach from phenotypes to systems medicine. Allergy 2011; 66: 596-604

22 Ballardini N, Bergström A, Wahlgren C-F, et al. IgE antibodies in relation to prevalence and multimorbidity of eczema, asthma, and rhinitis from birth to adolescence. Allergy 2016; 71: 342-49.

23 Baïz N, Slama R, Béné M-C, et al. Maternal exposure to air pollution before and during pregnancy related to changes in newborn's cord blood lymphocyte subpopulations. The EDEN study cohort. BMC Pregnancy Childbirth 2011; 11: 1-12.

24 Guxens M, Ballester F, Espada M, et al. Cohort Profile: The INMA-INfancia y Medio Ambiente-(Environment and Childhood) Project. Int J Epidemiol 2012; 41: 930-40.

25 Wijga AH, Kerkhof M, Gehring U, et al. Cohort profile: The Prevention and Incidence of Asthma and Mite Allergy (PIAMA) birth cohort. Int J Epidemiol 2014; 43: 527-35.

26 Pinart M, Benet M, Annesi-Maesano I, et al. Comorbidity of eczema, rhinitis, and asthma in IgE-sensitised and non-IgE-sensitised children in MeDALL: a population-based cohort study. Lancet Respir Med 2014; 2: 131-40.

27 Laprise C. The Saguenay-Lac-Saint-Jean asthma familial collection: the genetics of asthma in a young founder population. Genes Immun 2014; 15: 247-55.
28 Gref A, Merid SK, Gruzieva O, et al. Genome-wide interaction analysis of air pollution exposure and childhood asthma with functional follow-up. Am J Respir Crit Care Med 2017; 195: 1373-83.

29 Bonder MJ, Luijk R, Zhernakova DV, et al. Disease variants alter transcription factor levels and methylation of their binding sites. Nat Genet 2017; 49: 131-38.

30 McLean CY, Bristor D, Hiller M, et al. GREAT improves functional interpretation of cis-regulatory regions. Nat Biotechnol 2010; 28: 495-501.

31 Kundaje A, Meuleman W, Ernst J, Bilenky M, Yen A. Integrative analysis of 111 reference human epigenomes. Nature 2015; 518: 317-30.

32 Aguirre-Gamboa R, Joosten I, Urbano PCM, et al. Differential effects of environmental and genetic factors on $\mathrm{T}$ and $\mathrm{B}$ cell immune traits. Cell Rep 2016; 17: 2474-87.

33 Willer CJ, Li Y, Abecasis GR. METAL: fast and efficient meta-analysis of genomewide association scans. Bioinformatics 2010; 26: 2190-91.

34 Viechtbauer W. Conducting meta-analyses in $R$ with the metafor package. J Stat Softw 2010; 36: i03.

35 Paternoster L, Standl M, Chen C-M, et al. Meta-analysis of genome-wide association studies identifies three new risk loci for atopic dermatitis. Nat Genet 2012; 44: 187-92.

36 Reinius LE, Acevedo N, Joerink M, Pershagen G, Dahlen SE, Greco D Differential DNA methylation in purified human blood cells: implications for cell lineage and studies on disease susceptibility. PLoS One 2012; 7: e41361.

37 Tigchelaar EF, et al. Cohort profile: LifeLines DEEP, a prospective, general population cohort study in the northern Netherlands: study design and baseline characteristics. BMJ Open 2015; 5: e006772 (2015).

38 Schoenmaker M, et al. Evidence of genetic enrichment for exceptional survival using a family approach: the Leiden Longevity Study. Eur J Hum Genet 2006; 14: 79-84.

39 Willemsen G, et al. The Adult Netherlands Twin Register: twenty-five years of survey and biological data collection. Twin Res Hum Genet 2013; 16: 271-81.

40 George L, Brightling CE. Eosinophilic airway inflammation: role in asthma and chronic obstructive pulmonary disease. Ther Adv Chronic Dis 2016; 7: 34-51.

41 Astle WJ, Elding H, Jiang T, et al. The Allelic landscape of human blood cell trait variation and links to common complex disease. Cell 2016; 167: 1415-1429.e19.

42 Heinz S, Romanoski CE, Benner C, Glass CK. The selection and function of cell type-specific enhancers. Nat Rev Mol Cell Biol 2015; 16: 144-54.

43 Choy DF, Jia G, Abbas AR, et al. Peripheral blood gene expression predicts clinical benefit from anti-IL-13 in asthma. J Allergy Clin Immunol; 138: 1230-1233.e8.

44 Abdelsamed HA, Moustaki A, Fan Y, et al. Human memory CD8 T cell effector potential is epigenetically preserved during in vivo homeostasis. J Exp Med 2017; 214: 1593.

45 Youngblood B, Hale JS, Ahmed R. Memory CD8 T cell transcriptional plasticity. F1000Prime Rep 2015; 7: 38.

46 Vogel GF, Klee KMC, Janecke AR, Müller T, Hess MW, Huber LA Cargo-selective apical exocytosis in epithelial cells is conducted by Myo5B, Slp4a, Vamp7, and Syntaxin 3. J Cell Biol 2015; 211: 587.

47 Davies DE. Epithelial barrier function and immunity in asthma. Ann Am Thorac Soc 2014; 11: S244-51.

48 Frank SPC, Thon K-P, Bischoff SC, Lorentz A. SNAP-23 and syntaxin-3 are required for chemokine release by mature human mast cells. Mol Immunol 2011; 49: 353-58.

49 Shin J, Zhang P, Wang S, Wu J, Guan Z, Zhong X-P. Negative control of mast cell degranulation and the anaphylactic response by the phosphatase lipin1: Innate immunity. Eur J Immunol 2013; 43: 240-48.

50 Meana C, Peña L, Lordén G, et al. Lipin-1 Integrates lipid synthesis with proinflammatory responses during TLR activation in macrophages. J Immunol 2014; 193: 4614-22.

51 Levy BD. Sphingolipids and susceptibility to asthma. N Engl J Med 2013; 369: 976-78. 